\title{
Experiencias y aspiraciones educacionales de estudiantes de educación secundaria de distinta clase social en Valparaíso, Chile: similares aspiraciones, desiguales condiciones
}

Educational Experiences and Aspirations of Secondary School Students of Different Social Class in Valparaíso, Chile: Similar Aspirations, Unequal Conditions

Javier Donoso-Bravo'

\section{Resumen}

La presente investigación indagó las experiencias y aspiraciones educacionales y ocupacionales de secundarios pertenecientes a distintas clases sociales en la ciudad Valparaíso, con el propósito de avanzar hacia una mayor comprensión de la desigualdad socioeducativa en Chile. Basándonos en una metodología cualitativa, se realizaron grupos focales con estudiantes de clase popular, media y alta de acuerdo a la dependencia administrativa del colegio al que asistían. Los hallazgos nos muestran que los secundarios de las tres clases sociales construyen aspiraciones educativas y ocupacionales similares, a pesar de haber vivido diferentes experiencias socioeducativas de acuerdo a su origen social. Finalmente, se discute el alcance del concepto de clase social para el estudio de la desigualdad socioeducativa.

\section{Palabras clave}

Clase social, experiencia escolar, aspiraciones educacionales, secundarios.

\section{Abstract}

This research looked into the educational experiences and aspirations belonging to high school students of different social classes in the city of Valparaíso, aiming to reach a better comprehension of socio-educational inequality in Chile. Based on a qualitative methodology, we formed several focus groups with working, middle and upper class students according to the type of school they attended. Our findings show that high school students from the three social classes build up similar educational and occupational aspirations despite their different socioeducational experiences relative to their social origin. Finally, we discuss the utility and scope of the social class concept in the study of socioeducational inequality.

\section{Keywords}

Social class, educational experience, educational aspirations, high school students,

\section{Cómo citar/Citation}

Donoso-Bravo, Javier (2021). Experiencias y aspiraciones educacionales de estudiantes de educación secundaria de distinta clase social en Valparaíso, Chile: similares aspiraciones, desiguales condiciones. Revista de Sociología de la Educación-RASE, 14 (2), 171-189. http://dx.doi. org/10.7203/RASE.14.2.16667. 


\section{Introducción}

Actualmente, la educación —en términos de su formato institucionalizado a través de credencialesconstituye un recurso crucial para alcanzar ocupaciones que posibiliten la movilidad social ascendente o, al menos, la conservación de una determinada posición en la estructura social. Sin embargo, la obtención de credenciales educativas y, por ende, las oportunidades de vida que entregan, no se distribuyen de acuerdo a las capacidades y habilidades innatas de los individuos, sino que dependen sobretodo del origen social de los individuos, socavando así los principios meritocráticos que, se supone, están a la base de las sociedades occidentales modernas (Bourdieu y Passeron, 2003).

La desigualdad educativa es un fenómeno de amplia importancia en la actualidad, y ha sido investigado desde diversos ángulos: resultados educativos, acceso a la universidad, estratificación social del sistema de educación superior, traspaso de las credencias educativas a las oportunidades ocupacionales, entre otros. En todos estos aspectos, la desigualdad educativa en relación al origen social ha sido largamente evidenciada (Bourdieu y Passeron, 2003; Ruiz Herrero, 2011; Puga, 2011; Shavit y Blossfeld, 1993)

Este estudio se inscribe en esta tradición y busca poner de relieve las manifestaciones de la desigualdad social o de clase en las experiencias y aspiraciones educativas de secundarios de distinto origen social en la ciudad de Valparaíso en Chile, con el objetivo de avanzar en la comprensión de las desigualdades socioeducativas a partir de distintos contextos socioeducativos.

\section{La desigualdad socioeducativa: datos de contexto}

Durante la década de los 60, Bourdieu y Passeron (2003) mostraron que la diferencia en el logro educativo estaba asociada fundamentalmente a la desigual distribución del capital cultural entre las distintas clases sociales: los estudiantes de clase alta —y también de clase media - con un mayor volumen de capital cultural, obtenían mejores resultados académicos que sus pares de clase popular, y alcanzaban de esta manera mejores posiciones ocupacionales, reproduciéndose de esta manera la estructura social.

Posteriormente, diversos estudios han reafirmado el efecto de los factores culturales, sociales y económicos sobre el logro educativo de los individuos (García y Weiss, 2017; Lauer, 2003; Müller y Karle, 1993; Ruiz Herrero, 2011; Shavit y Blossfeld, 1993; Sieben et al., 2001; Tejedor y Caride, 1988; Treiman y Yip, 1989). En esa línea, la clase social se convierte entonces en una categoría fundamental para saber hasta dónde llegará un estudiante de acuerdo a la siguiente fórmula: a mayor nivel socioeconómico, mayor logro académico. Esto resulta problemático en la medida que el logro educacional está fuertemente relacionado con las oportunidades que encuentran los individuos a lo largo de su vida, de modo que, si las credenciales educativas se distribuyen esencialmente de acuerdo al origen social, nos encontraríamos con una sociedad con predominio de los factores adscriptivos en las opciones de movilidad social.

En el caso de Chile, la relación entre origen social y logro educativo también ha mostrado ser significativa tanto en el rendimiento escolar durante la educación media ${ }^{2}$ (Farías y Carrasco, 2012) así como en los resultados obtenidos por los estudiantes en la Prueba de Selección Universitaria (PSU) 3 (Muñoz y Redondo, 2013). Sin embargo, la desigualdad socioeducativa en Chile va mucho más allá del mero logro educativo.

\footnotetext{
2 Se usará indistintamente el calificativo de educación media o secundaria.

3 La Prueba de Selección Universitaria o PSU es un test estandarizado escrito, implementado en Chile desde 2003 para el proceso de admisión a las universidades tradicionales.
} 
El sistema educativo chileno básico y medio ${ }^{4}$ se compone de cuatro tipos de colegios según su dependencia administrativa ${ }^{5}$ : [a] colegios municipales: instituciones educativas administradas por los municipios, cuyo financiamiento depende en parte de éstos y del Estado, el cual entrega subvenciones en relación a la cantidad y asistencia de alumnos a clases, [b] colegios particulares subvencionados: instituciones educativas administradas por agentes privados (sostenedores) que reciben parte de su financiamiento a través de subvenciones estatales —equivalentes a las de los colegios municipales - y el resto de parte de las propias familias y/o apoderados de los estudiantes, mecanismo denominado copago, [c] colegios particulares pagados: instituciones educativas privadas que se autofinancian a través del pago de aranceles costeados por las propias familias y/o apoderados de los estudiantes y [d] colegios de corporaciones de administración delegada: instituciones educativas administradas por corporaciones y fundaciones sin fines de lucro (ligadas a organizaciones empresariales y productivas) que reciben una subvención estatal al igual que los colegios municipales y particulares subvencionados.

Otra clasificación relevante de los establecimientos educacionales en Chile tiene que ver con la modalidad de enseñanza que se imparte a partir de la educación secundaria, la que se divide en [a] científico-humanista, orientada a la profundización en áreas de formación general y [b] técnico-profesional, orientada a la formación en especialidades relacionadas con diferentes sectores económicos.

Estas clasificaciones no son sólo de carácter administrativo o formativo, sino que también social. La dependencia administrativa de los establecimientos educacionales en Chile están altamente correlacionados con el nivel socioeconómico de sus estudiantes: los colegios municipales —en su gran mayoría gratuitos - son los que reciben mayormente a los estudiantes de clase popular; los colegios particulares subvencionados — con un cierto rango de costo económico - corresponden al espacio educativo de la clase media, donde además de ciertas ventajas educativas, los estudiantes encuentran ventajas no educacionales que resultan significativas para el logro ocupacional, tales como capital social (Bellei, 2007; Puga, 2011); y por último, los colegios particulares pagados — los más costosos del sistema— corresponden al espacio educativo de la élite, y son los que muestran los mejores resultados en las pruebas estandarizadas de carácter nacional ${ }^{6}$.

La clasificación de acuerdo a la modalidad científico-humanista o técnico-profesional del establecimiento educacional también tiene una expresión social. La enseñanza técnico-profesional es la que recibe a los estudiantes de más bajos recursos del país, los que además muestran un bajo desempeño académico en comparación con sus pares de la modalidad científico-humanista durante la enseñanza media y, como consecuencia, tienen menores oportunidades de acceder a la educación superior (Farías y Carrasco, 2012).

Si bien la enseñanza superior se ha expandido de forma significativa durante las últimas dos décadas, no ha habido un acceso equitativo a estas nuevas oportunidades educacionales. Los estudiantes de origen popular que logran acceder a la enseñanza superior tienden a concentrarse en la educación técnica, mientras que aquellos de mayores recursos acceden mayoritariamente a carreras profesionales universitarias, las que - en términos generales - otorgan mejores perspectivas ocupacionales y económicas (Canales, 2016; Gaete y Morales, 2011). Esta segregación socioeducacional, por tanto, no sólo se expresa en el rendimiento educativo de los secundarios, sino que también conduce a un desigual aprovechamiento de las

\footnotetext{
4 Estos dos niveles corresponden a la enseñanza obligatoria en Chile.

5 Actualmente se encuentra en proceso gradual de aplicación la Ley de Inclusión Escolar, la cual va a modificar algunas de las características de este tipo de instituciones.

6 La OCDE (2004) ya había calificado al sistema educacional chileno como «conscientemente» dividido en clases sociales.
} 
oportunidades educativas y, por ende, a trayectorias educacionales y sociales distintas, contribuyendo de esta manera a la reproducción de la estructura social.

Esta desigualdad socioeducativa ha sido puesta ampliamente en evidencia a partir de puntajes, indicadores y cifras, sin embargo, ha sido menos explorada desde su manifestación subjetiva, es decir, desde las experiencias y aspiraciones de los propios estudiantes en sus contextos sociales específicos. En ese sentido, una aproximación subjetiva a esta desigualdad nos permite reconstruir la forma cómo se configuran las oportunidades objetivas y subjetivas para los individuos (Dubet y Martuccelli, 2000; Shavit et al., 2007; Willis, 2008).

Sepúlveda (2014) ha estudiado las trayectorias y experiencias de estudiantes de $4^{\circ}$ año medio —último año de secundaria - en el Gran Santiago y ha encontrado que la mayoría de los estudiantes sin importar su origen social o modalidad de estudio aspira a ingresar a la universidad y obtener un título profesional. Sin embargo, de acuerdo al autor, los estudiantes evaluarían la posibilidad de concreción de estas aspiraciones en relación a su realidad educativa y socioeconómica objetiva, lo que los llevaría a desplegar diferentes racionalidades en función de lo que ellos creen que podrían lograr.

La realidad socioeducacional en otras ciudades distintas a Santiago ha sido poco explorada. La ciudad de Valparaíso — una de las más importantes de Chile— constituye un caso particular, puesto que si bien es una «ciudad universitaria» — con un alto número de instituciones de educación superior tales como universidades, institutos profesionales y centros de formación técnica- muestra una marcada diferencia en el acceso a este nivel educativo según el origen social de los estudiantes: para 2017 sólo el 43\% de los estudiantes de colegios municipales egresados el año anterior logró matricularse en alguna de estas instituciones, frente al 57\% y $68 \%$ de los estudiantes de colegios particulares subvencionados y particulares pagados, respectivamente ${ }^{7}$. De hecho, si consideramos la modalidad de enseñanza media y el acceso a la universidad, los datos son aún más elocuentes: sólo el 13\% de los estudiantes de colegios municipales técnico-profesionales ingresó a la universidad en el año 2017. Por otro lado, el 44\% y 63\% de los estudiantes de colegios particulares subvencionados y particulares pagados (modalidad científico-humanista), respectivamente, se matriculó en la universidad el mismo año.

En este contexto de desigualdad social en las oportunidades educativas, indagamos en las experiencias y aspiraciones educacionales de estudiantes de enseñanza media de distintas clases sociales, con el propósito de avanzar hacia una mejor comprensión de la desigualdad socioeducativa en Valparaíso y Chile.

\section{Marco Conceptual}

Si bien en torno al significado y alcances del concepto clase social no existe un acuerdo generalizado, en términos generales refiere a las divisiones existentes en la sociedad de acuerdo a condiciones sociales y económicas. Típicamente, los individuos que pertenecen a la misma clase social comparten un nivel similar de riqueza, nivel educacional, tipo de trabajo o ingreso (Kerbo, 2003). Para Weber (1969) la clase se define en relación a una posición económica, la cual delimita un conjunto de oportunidades de vida y circunstancias para el conjunto de individuos que comparte esta posición.

\footnotetext{
Elaboración propia en base a datos del Ministerio de Educación (Mineduc) que corresponden a la cohorte 2016, que se matriculó en alguna institución de educación superior en 2017.
} 
Para Bourdieu (1989, p. 30) el sistema de clases sociales corresponde a «conjuntos de agentes ocupando posiciones similares que, situados en condiciones similares y sometidos a condicionamientos similares, tienen todas las probabilidades de tener disposiciones e intereses similares, luego de producir prácticas similares y parecidas tomas de posición». De esta manera, condiciones objetivas similares en la estructura social conducen a experiencias comunes del mundo social y, por lo tanto, llevan a experiencias subjetivas similares, lo cual nos permite hablar de un habitus de clase (Bourdieu, 1988). Este concepto refiere a las disposiciones y percepciones internalizadas por los sujetos en virtud de su clase social y, en ese sentido, permite dar cuenta de valores, creencias y experiencias compartidas con base a esta pertenencia social (Bourdieu, 2013). Sin embargo, esto no significa que individuos pertenecientes a la misma clase social sean iguales, sino que existen sistemas de disposiciones individuales como variantes estructurales de una socialización más o menos común que puede identificarse en cada individuo perteneciente a una determinada clase (Bourdieu, 1989).

La clase social es una categoría que cruza variados ámbitos de la vida social de los individuos, siendo especialmente relevante en el caso de la educación, por cuanto se ha demostrado su gran influencia sobre las diferencias en el logro académico de los estudiantes de acuerdo a su mayor o menor posesión de capital cultural, esto es, recursos objetivos y disposiciones subjetivas internalizadas por el individuo y que se transforman en un hábito o ethos más o menos favorable hacia la educación (Bourdieu, 1987). Los conceptos de capital cultural y habitus de clase, nos permiten adentrarnos más a fondo en el proceso socioeducativo de los individuos indagando en la dimensión cualitativa involucrada en éste, a la cual podemos aproximarnos a partir de sus experiencias, valores y aspiraciones educativo-ocupacionales en el marco de un sistema de clases.

Además de este marco, es necesario considerar una serie de categorías, las cuales — mediadas por la clase social— ofrecen un alcance explicativo medio:

[a] Factores institucionales: relacionados al papel organizativo y pedagógico de las instituciones educativas (Reay, David y Ball, 2001).

[b] Factores relacionales: asociados al papel de los otros significativos en torno a la educación (Hedström, 2005).

[c] Factores expresivos: relacionados con las experiencias y vivencias escolares de los estudiantes (Dubet y Martucelli, 2000).

Las disposiciones educativas no se despliegan en un contexto de clases rígido y compartimentado, sino que en un marco de relaciones culturales y simbólicas entre clases que señalan distinciones y/o dominaciones culturales, en la medida que «los sujetos sociales expresan y, al mismo tiempo, constituyen, para sí mismos y para los otros, su posición en la estructura social» (Bourdieu, 2002: 131).

Este sistema de clases es relacional, y en él se configuran diversas relaciones culturales y simbólicas entre las distintas clases. La cultura dominante alude a los bienes, valores o símbolos culturales que la clase social en la cima de la jerarquía logra imponer sobre las demás clases de la estructura social, las cuales internalizan éstos a partir de un proceso de asimilación cultural (Bourdieu, 2002). Desde una perspectiva complementaria, podemos entender el fenómeno a partir de otro concepto bourdesiano, histéresis del babitus, el cual refiere a la capacidad de permanencia y de soportar experiencias contradictorias por parte 
del habitus y volver a su estado original (Bourdieu, 1988). En un sentido similar, Lehmann (2014) habla de dislocación de habitus asociada a la transformación de los valores y actitudes tradicionalmente asociadas a una determinada clase social. Estos conceptos apuntan a comprender la posibilidad y alcances de los cambios de un determinado habitus.

Otra relación que se establece entre las clases corresponde a la de distinción social, a partir de la cual una determinada clase busca diferenciarse de otra u otras con la posesión, consumo o exhibición de ciertos bienes o símbolos que definen su posición en la estructura social y, al mismo tiempo, refuerzan su estatus. En ese sentido, la apropiación de algún arte, el consumo de ciertos alimentos o la posesión de determinado título universitario pueden operar en función de esta distinción social y simbólica perseguida fundamentalmente por las clases altas (Bourdieu, 1988).

\section{Método}

A partir de un enfoque cualitativo se buscó indagar las experiencias, valores y aspiraciones educativo-ocupacionales de estudiantes de $4^{\circ}$ año de enseñanza media de la ciudad de Valparaíso. Este nivel educativo corresponde al último año de la secundaria, el cual marca el fin de la enseñanza obligatoria y, por tanto, pone a los estudiantes ante importantes decisiones que tomar respecto a su futuro. En la medida que el camino que sigan va a determinar sus opciones ocupacionales, y, por ende, sus posibilidades de movilidad social, los estudiantes actúan como portavoces de las visiones, valores y aspiraciones de sus respectivas familias y clase social.

La problemática se abordó a partir de una metodología cualitativa, en la medida que se presenta más adecuada para adentrarse en las subjetividades de los individuos, en cómo experimentan la realidad social (Taylor y Bogdan, 1986), en este caso, las experiencias y aspiraciones educativo-ocupacionales de los estudiantes. La técnica de recolección de datos fue el grupo focal, cuya naturaleza grupal nos permitió conocer los discursos que elaboran los estudiantes, los cuales nos permitieron reconstruir los significados y sentidos que éstos le otorgan a su realidad educativa y social, la cual representa, al mismo tiempo, una experiencia de clase (Mella, 2000).

La selección de los casos se realizó a través de un muestreo de casos homogéneos (Patton, 2002) a partir del cual se utilizó la dependencia administrativa de los colegios — es decir, si corresponden a colegios municipales, particulares subvencionados o particulares pagados — para lograr homogeneidad en cuanto al origen de clase de los estudiantes que asisten a estos distintos tipos de establecimientos educacionales. Asociamos la pertenencia de clase de los estudiantes al tipo de colegio que asisten apoyándonos en estudios que demuestran la marcada estratificación institucional del sistema educacional en función de la clase de origen de los estudiantes (García-Huidobro, J. E., 2007; Farías, M., \& Carrasco, R., 2012).

Entre el segundo semestre de 2017 y el primero de 2018 se llevaron a cabo seis grupos focales: 3 en colegios municipales técnico-profesionales, 2 en colegios particulares subvencionados y 1 en un colegio particular pagado, en los cuales participaron de entre 6 a 8 estudiantes con representación mixta en términos de sexo. Estos se distribuyeron de acuerdo a cómo se señala en la Tabla I. 
Tabla I. Características grupos focales

\begin{tabular}{lcccc}
\hline $\begin{array}{c}\text { COLEGIO SEGÚN DEPENDENCIA } \\
\text { ADMINISTRATIVA }\end{array}$ & CLASE & N $^{\circ}$ DE GRUPO & N $^{\circ}$ PARTICIPANTES & $\begin{array}{c}\text { DISTRIBUCIÓN } \\
\text { POR SEXO }\end{array}$ \\
\hline \multirow{3}{*}{$\begin{array}{l}\text { SOCIAL } \\
\text { Colegios municipales }\end{array}$} & Clase popular & 1 & 6 participantes & 2 hombres $/ 4$ mujeres \\
& & 2 & 8 participantes & 4 hombres $/ 4$ mujeres \\
\hline \multirow{2}{*}{ Colegios particulares-subvencionados } & Clase media & 3 & 8 participantes & 3 hombres $/ 4$ mujeres \\
\hline Colegios particulares & & 1 & 6 participantes & 3 hombres $/ 3$ mujeres \\
\hline
\end{tabular}

Fuente: elaboración propia.

A partir de la utilización del programa para análisis cualitativo asistido por computadora Atlas.ti 7, se llevó a cabo un Análisis de Discurso en la medida que se buscó analizar los discursos para leer la realidad social, entendiendo que los mismos participan y tienen injerencia en la constitución de la realidad social (Fairclough, 1995), en este caso, la realidad socioeducativa estudiada. A partir de este tipo de análisis se organizaron los datos agrupándolos en 'matrices de sentido' relativas a las categorías de análisis con el fin de conocer el significado latente involucrado en los discursos de los estudiantes.

\section{Hallazgos}

\subsection{Experiencias educacionales desiguales en relación a la enseñanza superior}

Los grupos focales realizados con los estudiantes de educación media de Valparaíso pertenecientes a distintas clases sociales nos permitieron indagar en su experiencia de esta etapa educativa tanto en su preparación para la educación superior como para el trabajo. En ese sentido, la experiencia vivida por los estudiantes durante su enseñanza media está estrechamente ligada a su condición de clase: una experiencia que es propia de los estudiantes de clase popular, y otra para los estudiantes de clase media y alta.

Como ya señalamos anteriormente, los colegios municipales en su modalidad técnico-profesional son los establecimientos que reciben a gran parte de los estudiantes de origen popular, sin embargo, este hecho no se deriva de una decisión racional de éstos o sus familias, sino más bien se relaciona a una imposición derivada de su posición social. La posibilidad de acceder a un colegio particular subvencionado o particular pagado para las familias de bajos recursos es bastante limitada —especialmente por motivos económicos, aunque también académicos ${ }^{8}$ - por lo que los colegios municipales técnico-profesionales acaban siendo la única posibilidad para que sus hijos continúen sus estudios secundarios.

Al finalizar el $2^{\circ}$ año medio, los estudiantes de colegios municipales técnico-profesionales deben optar por alguna de las especialidades técnicas que ofrecen estos colegios, de modo que, durante el $3^{\circ}$ y $4^{\circ}$ año de la enseñanza media los estudiantes reciben una formación orientada principalmente hacia una especialidad técnica, y como contraparte, menos conocimientos preparatorios para la educación superior, lo cual es advertido por los propios estudiantes de estos colegios.

8 Actualmente se encuentra en curso la aplicación de la Ley de Inclusión, la cual —entre otros aspectos— busca acabar con la selección de estudiantes por motivos académicos en colegios municipales y particulares subvencionados. 
«Para los que pretenden otra carrera igual es malo porque no tiene el piso minimo para entrar a la $U^{9}$, de hecho, es muy diferente, muy atroz. la diferencia que hay entre esto y la U». (Mujer, colegio municipal técnico-profesional, clase popular).

"Vamos atrasados en materia. De hecho, hay materia de cuarto... Hay materias muy diferentes, porque igual yo voy a un programa los sábados y es muy... lo que pasan en matemáticas yo quedo asi muy 'colgada', muy 'colgada"10. Y dicen: 'no, esto es lo más básico que pueden saber'. Y no lo he visto. Es como malis. (Mujer, colegio municipal técnico-profesional, clase popular).

Los estudiantes reconocen que esta insuficiencia de conocimientos los pone en una situación de desventaja en relación a sus pares de otros tipos de colegios en cuanto a sus posibilidades de acceso a la educación superior, sobre todo a la universidad. Por su lado, los estudiantes de clase media y alta, quienes se concentran en colegios particulares subvencionados y particulares pagados, respectivamente, tienen una experiencia radicalmente opuesta a la de los estudiantes de clase popular, puesto que la configuración de su espacio educativo, está rigurosamente diseñado para prepararlos para la enseñanza superior, especialmente, para la universidad.

Para los estudiantes de clase media y alta el ingreso a la universidad constituye una obligación social y, por tanto, la etapa de la educación media — especialmente, el último año- es vivida como un periodo de preparación para aquélla.

«Aquí también nos fortalecemos en el ámbito educacional, o sea, igual en el colegio nos preparan para enfrentarnos a lo que es la universidad») (Hombre, colegio particular subvencionado, clase media).

"Yo encuentro que... no sé si será asi en otros colegios, pero al menos acá yo siento que nos han enseñado todo desde el punto de vista de las materias, de saber cosas para que nos vaya bien en lo que viene». (Hombre, colegio particular, clase alta).

Esta preparación tiene uno de sus hitos más significativos en la rendición de la Prueba de Selección Universitaria (PSU), examen estandarizado de admisión a la universidad que rinden los estudiantes que finalizan la enseñanza media para acceder a las universidades tradicionales ${ }^{11}$. En este sentido, si bien tanto los estudiantes de clase media y alta reciben una formación orientada a lograr un puntaje que les permita ingresar a la carrera universitaria que aspiran, los primeros creen no haber tenido una preparación del todo satisfactoria por parte de sus colegios.

«Toda la materia que pasaron como que fue en vano casi, porque se ve muy poco de lo que realmente se debería ver para la PSU». (Hombre, colegio particular subvencionado, clase media).

9 El término «la U» se refiere de forma genérica a la Universidad.

10 Término coloquial que se refiere a quedar sin entender una conversación o situación.

11 Las universidades tradicionales son aquellas creadas antes de la reforma universitaria de 1981 o que se derivaron de éstas con la misma reforma. Estas universidades son las más prestigiosas y se agrupan en el Consejo de Rectores de Chile. Actualmente hay algunas universidades no tradicionales que se han incorporado al Sistema Único de Admisión a través de PSU pero aun así no forman parte del Consejo de Rectores. 
«Para poder llegar a la universidad necesitamos pasar por una prueba de aptitud (...) y la educación que nos dan aqui no nos sirve mucho para esa prueba». (Mujer, colegio particular subvencionado, clase media).

Más allá de la evaluación crítica que hacen los estudiantes de clase media de su preparación para la PSU, hay un ámbito en el que su experiencia se asemeja a la de sus pares de clase alta: la presión familiar por conseguir un puntaje PSU que les permita acceder a una universidad tradicional. En ese sentido, son los padres los principales promotores de que sus hijos vayan a la universidad, y con este fin, elaboran un exigente programa académico — especialmente en el último año de enseñanza media — el cual, además de la formación regular impartida por el colegio, incluye la asistencia a pre-universitarios (instituciones extra-escolares que preparan específicamente para la PSU) y, a veces, clases particulares.

\begin{abstract}
«Y por eso uno muchas veces tiene que aparte de estudiar, estar en un pre [preuniversitario], e igual te toma mucho tiempo porque uno no alcanza a hacer nada, llega tarde a la casa, sale del colegio, va al preuniversitario, llega tarde a la casa, entonces no tiene tiempo como de hacer nada». (Hombre, colegio particular subvencionado, clase media).
\end{abstract}

«Ahora encuentro que la educación en sí está como muy ... no sé... muy mal llevada, porque pasamos como todo... tenemos una jornada extensa y después llegamos a la casa a estudiar de nuevo». (Mujer, colegio particular, clase alta).

Esta sobrecarga académica experimentada por estos estudiantes se asocia directamente con sus respectivos habitus de clase media y alta, en los cuales la educación universitaria constituye un objetivo crucial si se pretende ascender o conservar la posición en la estructura social. En este sentido, si bien los estudiantes de clase alta cuentan con la posibilidad de cursar su enseñanza secundaria en colegios particulares privados, instituciones de élite que son las que obtienen los mejores puntajes en las pruebas estandarizadas, la clase media — sin el capital económico para acceder a ese tipo de colegiosenvía a sus hijos a colegios particulares subvencionados, los que además de mostrar resultados en promedio más altos que los colegios municipales, tienden a concentrar a los estudiantes pertenecientes a esta misma clase y, por lo tanto, compartiendo experiencias con pares de pertenencia social similar.

La presión sobre los estudiantes de clase media por obtener un puntaje PSU que les permita acceder a una carrera en una universidad tradicional, y de esta manera, una mejor perspectiva de futuro, lleva a que esta prueba sea vivida por los estudiantes como una instancia prácticamente definitoria para sus vidas. En este contexto, emerge un discurso fuertemente crítico hacia este examen. 
«El problema es que la PSU no debería ser...no es el método más apropiado para medir la capacidad del estudiante, ya que tener ochocientos cincuenta [de puntaje] de PSU'12, en todas las que rindas, no significa necesariamente que te va a ir bien en la universidad, de hecho, es quizá más una prueba como de memoria o de cosas que pudiste aprender en el colegio, pero no significa tu capacidad para poder sobrevivir en el mundo laboral o en el mismo mundo universitario, entonces algo tiene... algo necesita para ser más buena para medir al estudiante». (Hombre, colegio particular subvencionado, clase media).

«Es lo que yo pienso... que estudiamos tanto para después medirnos por una prueba para entrar a la universidad, tenemos que estar metiéndonos a un pre-universitario para entrar a la universidad, en vez. de medirnos los años de esfuerzo que tenemos todos desde chicos hasta que terminamos». (Mujer, colegio particular subvencionado, clase media).

Más allá de la visión crítica sostenida por los estudiantes de clase media respecto a la PSU, su pertenencia a colegios particulares subvencionados los hace parte de un ordenamiento institucional educativo o habitus institucional (Reay, David y Ball, 2001) — del cual también forman parte los estudiantes de clase alta en los colegios particulares pagados - que los prepara para esta prueba y para la universidad no sólo a través de la entrega de conocimientos específicos sino también a partir de una serie de actividades extra-académicas que buscan que los estudiantes alcancen el objetivo universitario satisfactoriamente: charlas y test vocacionales, participaciones en ferias vocacionales fuera y dentro del colegio, programas de reforzamiento, ensayos para la PSU, entre otros.

Por su lado, los colegios municipales técnico-profesionales, que forman principalmente a los estudiantes de clase popular, están orientados a entregarles a éstos una formación de nivel medio en alguna especialidad que les permita desempeñarse laboralmente en algún sector productivo de la ciudad o la región. De esta forma, el habitus institucional de este tipo de colegios no pretende que sus estudiantes accedan a la universidad — ni tampoco a la enseñanza técnica superior — su propósito primordial es que cuenten con una credencial que los habilite para el mundo laboral una vez que terminen la educación media. Los estudiantes desconocen las características de las instituciones de educación superior, los contenidos de la PSU, los plazos y requisitos de acceso a la universidad, las vías de financiamiento, lo cual refleja no sólo un bajo capital cultural adquirido familiarmente, sino que también un habitus institucional no proclive a los estudios superiores por parte de los establecimientos municipales técnico-profesionales.

La institución escolar mediatiza las condiciones materiales de vida junto con el capital cultural de entrada, lo que genera trayectorias y experiencias distintas en relación al origen social de los estudiantes. Mientras los estudiantes de clase media y alta son preparados intensamente para la universidad, sus pares de clase popular ya han tenido sus primeras experiencias en el mundo laboral a través de las prácticas en empresas que han debido realizar como parte de su propio programa formativo y/o por trabajos realizados de manera extra-curricular. En este sentido, los estudiantes de origen popular se encuentran tempranamente vinculados al mundo del trabajo no solo a causa de la modalidad formativa que cursan, sino que también por sus propias condiciones socioeconómicas, las que los empujan muchas veces a tener que generar ingresos por su parte. Estas experiencias disímiles se relacionan con

12 Sobre 800 puntos en la PSU se considera un resultado sobresaliente. 
el concepto de 'experiencia escolar' de Dubet y Martuccelli (2000) el cual nos permite observar estas experiencias sociales diversas de los estudiantes no sólo como reflejo de una condición social sino que también a partir de ellos mismos construyendo su experiencia y a sí mismos en un contexto socioescolar determinado.

En suma, los estudiantes de clase popular, por un lado, y los de clase media y alta, por otro, tienen experiencias visiblemente opuestas de la enseñanza secundaria en la ciudad de Valparaíso. Mientras los primeros se desarrollan en un medio familiar, social e institucional menos proclive hacia las prácticas relacionadas con la cultura académica, lo cual los aleja progresivamente de la universidad, sobretodo de las instituciones más prestigiosas; los segundos, en condiciones objetivas más favorables, se desarrollan en un entorno social, familiar y educativo que les provee y potencia el capital cultural necesario para una trayectoria con mayores posibilidades de éxito hacia la educación superior, y sobre todo, a la universidad.

\subsection{Aspiraciones educativo-ocupacionales: entre la asimilación y la distinción}

Históricamente en las sociedades occidentales, la universidad ha sido dominio exclusivo de las élites. En América Latina las primeras universidades aparecieron en el siglo XVI durante la colonización española y por siglos constituyeron patrimonio exclusivo de las clases acomodadas, para recién durante el siglo xx hacerse accesibles para las clases medias. Por otro lado, las clases populares estuvieron largamente marginadas de las universidades.

Las primeras explicaciones de la nula o escasa participación de las clases populares en la universidad, se atribuyó a sus limitadas aspiraciones educativas y ocupacionales (Hyman, 1953). En esa línea, se evidenció que los estudiantes hombres de origen popular aspiraban a ocupaciones manuales por la influencia que ejercía el hecho de que sus padres llevaran a cabo estas mismas ocupaciones (Raby \& Caldwell, 1981), y también por la existencia de una 'cultura masculina' en torno a las ocupaciones de tipo manual, la que orientaba a los estudiantes a este tipo de trabajos (Willis, 1988).

En Chile, a pesar de que la cobertura de la enseñanza universitaria ha tenido una notable expansión en las últimas dos décadas, las clases medias son las que más se han beneficiado a partir del acceso de sus miembros que anteriormente no lograban ingresar a la universidad, quedando los estudiantes de origen popular aun ampliamente marginados de la misma. Actualmente, sin embargo, la baja presencia de estos estudiantes es difícilmente atribuible a limitadas aspiraciones educativo-ocupacionales, puesto que al igual que sus pares del Gran Santiago (Sepúlveda, 2014), los secundarios de origen popular de la ciudad de Valparaíso también aspiran a llegar a la universidad y desempeñar ocupaciones profesionales.

Los estudiantes de clase popular son conscientes de que completar la enseñanza media técnico-profesional les ofrece limitadas opciones laborales, y escasas posibilidades de movilidad social, de esta manera, la continuación de estudios superiores aparece como la única posibilidad de alcanzar mejores condiciones económicas a futuro. Estas aspiraciones universitarias, sin embargo, trascienden las motivaciones meramente económicas y se entremezclan con el deseo de desarrollar una vocación. 
«Me encantan los niños y desde chica he estado con esa cuestión de estudiar para educadora diferencial, entonces uno empieza con sueños, y después cumplir la meta». (Mujer, colegio municipal técnico-profesional, clase popular).

«A mi más que la plata me incentiva que me gusta... me gusta estar entre números... por lo menos a mi me gusta estar entre números, me veo entre papeles, con responsabilidad, eso me motiva». (Mujer, colegio municipal técnico-profesional, clase popular).

Las crecientes aspiraciones educativas y ocupacionales de los estudiantes de clase popular reflejan la paulatina asimilación de la cultura académica instituida y promovida por la clase alta y media. En ese sentido, aunque los estudiantes de origen popular no se desenvuelven en un contexto familiar ni institucional proclive a la universidad, han internalizado esta cultura a partir de otras influencias.

«Yo veo por mis profesoras las del colegio, que son como las que más te incitan a ir a la u e ir alti$r^{13}$ a una ingeniería». (Mujer, colegio técnico-profesional, clase popular).

«En el colegio, yo creo que todos nosotros hemos adquirido conocimientos de la vida a través de los profesores». (Mujer, colegio técnico-profesional, clase popular).

«La mayoría de, por ejemplo, de parte de un lado de mi familia no tienen estudios superiores, pero pa'l otro quincho ${ }^{14}$, por decirlo así, mis tios, mis primos, son todos universitarios». (Mujer, colegio municipal técnico-profesional, clase popular).

Lehmann (2014) observa que, en el contexto de una masificación del sistema universitario, los jóvenes de clase popular acceden a nuevas experiencias, relaciones e informaciones que, al ampliar su conocimiento, les muestra un horizonte más variado de opciones educacionales, ocupacionales y vitales. En una perspectiva similar, Sellar y Gale (2011) señalan que las características de la modernización y globalización en el mundo contemporáneo han impactado en el desarrollo de la imaginación y el crecimiento de las aspiraciones de los individuos de clase popular, provocando una dislocación de su babitus de clase tradicionalmente asociado a ocupaciones manuales y poco calificadas.

Este proceso de asimilación de la cultura académica por parte de los estudiantes de clase popular se observa también en que éstos no sólo aspiran a llegar a la universidad, sino que además desean ingresar a universidades tradicionales, aquellas instituciones más selectivas, prestigiosas y exigentes del sistema.

«En las universidades públicas tú luchas para entrar, pero en las universidades privadas tú puedes entrar porque vai a pagar y te van a dejar igual porque estai pagando». (Hombre, colegio municipal técnico-profesional, clase popular).

13 Modismo chileno que se refiere al acto de realizar algo apresuradamente, rápidamente o en la inmediatez.

14 Término coloquial para referirse al otro lado de la familia (paterna o materna). 
"Si una persona de una universidad estatal (tradicional) y una privada van a pedirpega $a^{15}$ ¿a quién van a contratar? A la de la estatal, porque van a saber que se esforzó más que el de la universidad privada». (Hombre, colegio municipal técnico-profesional, clase popular).

Esta mayor valoración de las universidades tradicionales por parte de los estudiantes de clase popular tiene que ver con el mérito que ellos atribuyen a quienes logran ingresar a estas universidades y, con mayor razón, titularse. Por lo mismo, en la medida que un buen puntaje en la PSU constituye una condición indispensable para acceder a estas universidades, los estudiantes asumen que un buen resultado es muestra de inteligencia y capacidad.

\title{
«Las universidades son más exigentes, porque exigen puntaje PSU para entrar. En los institutos es más fácil, más accesible». (Mujer, colegio municipal técnico-profesional, clase popu- lar).
}

\begin{abstract}
"Hay una cuestión de inteligencia, ya que en los Centros de Formación Técnica e Institutos no te van a pedir puntaje PSU, solamente te van a pedir PSU rendida, la universidad, en cambio, te piden un puntaje para ingresar a una carrera». (Hombre, colegio municipal técnico-profesional, clase popular).
\end{abstract}

Esta apreciación, marcadamente opuesta a la de los estudiantes de clase media y alta — quienes son especialmente críticos de este examen- es reflejo de la distancia que tienen los estudiantes de clase popular con la cultura académica, es decir, un entorno socio-familiar menos proclive a la educación y más ajeno a la universidad —al menos, en comparación con sus pares de clase media y alta— lo que les dificulta la elaboración de un discurso crítico respecto a esta prueba.

La formación de este «nuevo» habitus en los estudiantes de origen popular, reflejado especialmente en sus aspiraciones universitarias, corresponde a un proceso de asimilación de la cultura dominante, la cual en este contexto se expresa a través de la universidad, destino anhelado por buena parte de estos estudiantes, y que ha sido alentado por el menosprecio que éstos advierten existe sobre las ocupaciones técnicas en la sociedad chilena.

\section{«La valoración también es un tema, porque aqui en Chile valoran más al universitario que al técnico». (Mujer, colegio municipal técnico-profesional, clase popular).}

«Mi otra parte de la familia, que todos... algunos están recién en el técnico y a algunos ya les pagan muy poco a diferencia que a mis tíos que ya son ingenieros». (Mujer, colegio municipal técnico-profesional, clase popular).

Si bien estos estudiantes valoran la formación técnica que han recibido en la medida que les ha provisto de herramientas para el trabajo, así como también una experiencia del mundo laboral, sus planes no consideran continuar estudios técnicos de nivel superior, lo cual parecería ser el camino más coherente de acuerdo a su trayectoria educacional. Por el contrario, los estudiantes aspiran a seguir

15 Modismo chileno referido a trabajo, profesión u oficio. 
carreras universitarias seguramente por la mayor valoración social que ellos advierten existe por parte de la sociedad.

Por otro lado, los estudiantes de clase media coinciden con sus pares de clase popular en cuanto a la baja consideración de las ocupaciones técnicas en la sociedad chilena. A pesar de eso, y aunque su apuesta mayor es ingresar a la universidad y desempeñar una ocupación profesional a futuro, acceder a una carrera técnica aparece como una alternativa posible para estos estudiantes.

«El técnico también saca un título y estudia menos años, entonces mejor estudio un técnico por menos años, que estar matándome cinco años para sacar un título profesional y no voy a trabajar en lo que yo me esforcé cinco años» (Mujer, colegio particular subvencionado, clase media).

"La verdad yo siempre tuve, asi como la universidad, la universidad, y que sea estatal, y todo eso. Pero ahora, ya queda tan poco para la PSU y viendo eso he estado averiguando sobre centros de formación técnica e institutos profesionales porque siento que no estoy preparado para dar la PSU, ni tampoco en todo el año me lo tomé... así como a conciencia, haciendo preuniversitario y todo eso (...) entonces por eso estoy buscando alterna... plan b». (Hombre, colegio particular subvencionado, clase media).

Estas opciones barajadas por los estudiantes son, sin embargo, rechazadas por sus padres, quienes los impulsan fuertemente hacia carreras universitarias, especialmente aquellas más prestigiosas. Sin embargo, esta estrategia pareciera no ser tan efectiva como antes en la medida que la devaluación de los títulos profesionales provocada por la ampliación de la cobertura universitaria ha tenido como consecuencia que este tipo de ocupaciones ya no ofrezcan el nivel de ingresos ni seguridad económica que aseguraban en el pasado; incluso, algunas ocupaciones técnicas gozan de un mayor nivel de empleabilidad e ingresos que algunas profesionales, hecho impensado décadas atrás.

Entonces, ¿por qué la clase media sigue apostando por las carreras profesionales y desechando las técnicas? El relativo declive económico de las ocupaciones profesionales no las ha despojado del reconocimiento y prestigio al cual han estado tradicionalmente asociadas, y del cual han gozado tanto la clase alta como media. En caso de que sus hijos no logren acceder a una universidad tradicional — reducto tradicional de la clase media - los padres prefieren que éstos se preparen para rendir nuevamente la PSU al año siguiente o ingresen a una universidad privada, pero que no estudien una carrera técnica, la que, aunque eventualmente podría ofrecerles buenas perspectivas económicas, se asocia a la clase popular y, por lo tanto, podría provocarles una fractura en su condición de clase. De esta forma, el comportamiento de esta clase en relación a la educación mantiene las características tradicionales del babitus de clase media, o lo que Brooks (2003) denomina clase media liminal, a partir de su tendencia a homegeneizarse culturalmente y distinguirse en sus aspiraciones y conductas de la clase obrera tradicional, al mismo tiempo, que busca emular a la clase alta en sus valores y pautas culturales (Bourdieu, 2002).

En una sociedad altamente estratificada como la chilena, los discursos de los estudiantes de distinto origen social nos han mostrado que tanto los estudios como la ocupación funcionan como una potente marca de clase, de ahí que, para los estudiantes de clase media, y también para los de clase alta, exista una trayectoria determinada a seguir, de la cual no se pueden desviar so pena de entrar en conflicto 
con sus progenitores, quienes fuertemente involucrados en su proceso educativo, procuran conservar o mejorar su estatus en la estructura social.

Las relaciones culturales que se establecen entre las clases sociales dan cuenta de una progresiva homologación entre los espacios simbólicos o habitus de las distintas clases, lo cual hemos advertido a partir de las aspiraciones educativas y ocupacionales de los secundarios de Valparaíso, sin embargo, éstas se configuran en el marco de condiciones objetivas y estructurales diferenciales que, sobre todo, constituyen un gran obstáculo para que los estudiantes de clase popular concreten sus crecientes aspiraciones.

\section{Conclusiones}

En el contexto social y educativo del Chile actual, las aspiraciones educativas y ocupacionales de los estudiantes de enseñanza media han tendido a homogeneizarse entre clases sociales, en la medida que el acceso a la universidad hoy constituye un gran anhelo para los estudiantes de diverso origen social, sobre todo en ciudades como Valparaíso, la cual como «ciudad universitaria» cuenta con una amplia oferta de instituciones de educación superior y universitarias. Sin embargo, las trayectorias sociales, familiares y educativas de los estudiantes nos revelan diferencias significativas de acuerdo al tipo de colegio que asisten y su origen de clase, lo cual tiene un gran impacto sobre la posibilidad de concretar esas aspiraciones.

Los estudiantes de clase popular — cuya mayoría asiste a colegios municipales técnico-profesionales - quieren llegar a la universidad, sin embargo, a lo largo de su trayectoria se enfrentan a una serie de barreras sociales, culturales e institucionales que tienden a alejarlos progresivamente de la universidad, por lo que sus aspiraciones se vuelven cada vez más difíciles de concretar. Por otro lado, los estudiantes de clase media y alta, más aventajados en cuanto a su preparación para la universidad producto de su posición social, al mismo tiempo, se ven sometidos a una fuerte presión familiar y social por acceder a la universidad y alcanzar una ocupación profesional derivada de la obligación de clase.

En este sentido, las clases sociales constituyen una categoría analítica fundamental no sólo para entender las experiencias y trayectorias educativas de los individuos, sino también para comprender las prácticas y relaciones culturales que han llevado, por un lado, a la valorización de determinadas opciones formativas y/u ocupacionales, tales como la universidad y las carreras profesionales, y, por otro, a la desacreditación de otras, como las carreras técnicas.

En el mismo sentido, cabe resaltar la relación entre el habitus de clase y el habitus institucional de los establecimientos educativos, en la medida que este último cumple un papel fundamental en propiciar a través de distintas instancias y dispositivos determinadas trayectorias educativo-ocupacionales, reforzando de esta manera los habitus de clase de los estudiantes que ya se vienen construyendo a partir de su origen social con la influencia de sus padres, familiares y pares antes y durante su período educativo. De esta forma, ambos habitus se van reforzando mutuamente a lo largo de la trayectoria de los estudiantes construyendo un babitus consolidado que va a ejercer una gran influencia al fin de la enseñanza media cuando los estudiantes deban tomar decisiones respecto a su futuro inmediato.

A partir de los hallazgos se vuelve necesario repensar la universidad en el contexto actual. Ante la inviabilidad que todos puedan acceder a la enseñanza universitaria, es imprescindible impulsar y promover diversas trayectorias formativas que no sólo sean útiles para la sociedad, sino que también puedan constituir proyectos vitales significativos para los propios individuos, y de esta manera, evitar la eventual 
desilusión y frustración para quiénes, situados en una posición de desventaja socioeducativa, encuentran más obstáculos para acceder a la universidad.

Lo anterior no significa que no sea relevante también avanzar en la disminución de la desigualdad en el acceso y permanencia en la universidad y la educación superior de acuerdo al origen social, por cuanto esta inequidad educativa se basa en desigualdades arbitrarias producidas por la propia estructura de clases, las cuales se arrastran desde la cuna y se reproducen a lo largo del tiempo. Esto es imprescindible si queremos avanzar hacia un sistema educativo que efectivamente se sostenga sobre principios meritocráticos y no adscriptivos.

\section{Referencias bibliográficas}

Bellei, Cristian (2007): "Expansión de la educación privada y mejoramiento de la educación en Chile. Evaluación a partir de la evidencia”. Revista Pensamiento Educativo, 40 (1), 285-311 (en línea). https:// biblat.unam.mx/es/revista, consultado el 5 de mayo de 2020.

Bourdieu, Pierre (2013). Argelia 60. Estructuras económicas y estructuras temporales. Buenos Aires: Siglo XXI.

Bourdieu, Pierre (2002): “Condición de clase y posición de clase”. Revista Colombiana de Sociología, 7 (1), 119-141 (en línea). https://revistas.unal.edu.co/index.php/recs/article/view/11153, consultado el 17 de noviembre de 2019.

Bourdieu, Pierre (1989): "El espacio social y la génesis de las 'clases”. Estudios sobre las Culturas Contemporáneas, 3 (7), 27-55 (en línea). http://culturascontemporaneas.com/contenidos/espacio_social_y_genesis.pdf, consultado el 5 de noviembre de 2019.

Bourdieu, Pierre (1988). La distinción. Criterios y bases sociales del gusto. Madrid: Taurus.

Bourdieu, Pierre (1987): “Los tres estados del capital cultural”. Sociológica, UAM-Azcapotzalco, (5), 11-17 (en línea). http:// sociologiac.net/biblio/Bourdieu-LosTresEstadosdelCapitalCultural.pdf, consultado el 13 de abril de 2019.

Bourdieu, Pierre y Passeron, Jean Claude (2009). Los herederos. Los estudiantes y la cultura. Buenos Aires: Siglo XXI.

Brooks, Rachel (2003): "Discussing Higher Education Choices: Differences and Difficulties". Research Papers in Education, 18 (3), 237-258. DOI: https://doi.org/10.1080/0267152032000107310.

Canales, Andrea (2016): "Diferencias socioeconómicas en la postulación a las universidades chilenas: el rol de factores académicos y no académicos". Revista Calidad en la Educación, (44), 129-157 (en línea). https:/ / www.cned.cl/sites/default/files/revista/cse_articulo1206.pdf, consultado el 3 de diciembre de 2020.

Dubet, Francoise y Martuccelli, Danilo (2000). En la escuela. Sociología de la experiencia escolar. Buenos Aires: Losada.

Fairclough, Norman (1995). Critical Discourse Analysis. London: Longman.

Farías, Mauricio y Carrasco, Rafael (2012): "Diferencias en resultados académicos entre educación técnico-profesional y humanista-científica en Chile". Calidad en la educación, (36), 87-121. DOI: https:// dx.doi.org/10.4067/S0718-45652012000100003. 
Gaete, Marcela y Morales, Raquel (2011): "Articulación del sistema de educación superior en Chile: posibilidades, tensiones y desafíos". Revista Calidad en la Educación, (35), 51-89. DOI: https://doi. $\operatorname{org} / 10.31619 /$ caledu.n35.96.

García, Emma y Weiss, Elaine (2017): "Education Inequalities at the School Starting Gate: Gaps, Trends and Strategies to Address Them". Economic Policy Institute (en línea). http://epi.org/132500, consultado el 2 de marzo de 2020.

García-Huidobro, Juan Eduardo (2007). "Desigualdad educativa y segmentación del sistema escolar. Consideración a partir del Caso Chileno”. Revista Pensamiento Educativo, 40 (1), 65-85 (en línea). http:/ / pensamientoeducativo.uc.cl/index.php/pel/article/view/399, consultado el 18 de julio de 2019.

Hedström, Peter (2005). Dissecting the Social. Cambridge: Cambridge University Press.

Hyman, Herbert (1972): "Los sistemas de valor de diferentes clases: Una contribución socio-psicológica al análisis de la estratificación” en Reinhard Bendix y Seymour M. Lipset (eds.): Clase, status y poder (vol. II). Madrid: Euramérica.

Kerbo, Harold (2003). Estratificación social y desigualdad. El conflicto de clase en perspectiva histórica y comparada. Madrid: McGraw Hill

Lauer, Charlotte (2003): "Family Background, Cohort and Education: A French-German Comparison Based on a Multivariate Ordered Probit Model of Educational Attainment". Labour Economics, 10 (2), 231-251. DOI: https://doi.org/10.1016/S0927-5371(03)00007-1.

Lehmann, Wolfgang (2014). "Habitus Transformation and Hidden Injuries. Successful Working-Class University Students”. Sociology of Education, 87 (1), 1-15. DOI: https://doi.org/10.1177\%2F0038040 713498777.

Mella, Orlando (2000). “Grupos focales ('focus groups'). Técnica de investigación cualitativa”. Documento de trabajo, CIDE, Santiago de Chile, (3), 1-27 (en línea). http:// files.palenque-de-egoya.webnode. es/200000285, consultado el 14 de abril de 2020.

Müller, Walter y Karle, Wolfgang (1993): "Social Selection in Educational Systems in Europe”. European Sociological Review, 9 (1), 1-23 (en línea). http:/ / www.jstor.org/stable/522823, consultado el 28 de mayo de 2020.

Muñoz, Pablo y Redondo, Amaia (2013): “Desigualdad y logro académico en Chile". Revista CEPAL, (109), 107-123 (en línea). https:/ / repositorio.cepal.org/handle/11362/11573, consultado el 1 de marzo de 2019.

OCDE (2004): "Revisión de políticas nacionales en educación: Chile. París y Santiago". OCDE y MINEDUC (en línea). http://www7.uc.cl/webpuc/piloto/pdf/informe_OECD.pdf, consultado el 20 de diciembre de 2018.

Patton, Michael Quinn (2002). Qualitative Research and Evaluation Methods. Thousand Oaks: Sage Publications. 
Puga, Ismael. (2011): "Escuela y estratificación social en Chile: ¿cuál es el rol de la municipalización y la educación particular subvencionada en la reproducción de la desigualdad social?” Estudios pedagógicos (Valdivia), 37 (2), 213-232. DOI: https://dx.doi.org/10.4067/S0718-07052011000200013.

Raby, Leo y Walford, Geoffrey (1981): “Career Related Attitudes and Their Determinants for Middleand Low-Stream Pupils in an Urban, Multiracial Comprehensive School”. Research in Education, (25), 19-35. DOI: https://doi.org/10.1177\%2F003452378102500103.

Reay, Diane; David, Miriam y Ball, Stephen (2001): "Making a Difference?: Institutional Habituses and Higher Education Choice”. Sociological Research Online, 5 (4), 1-12. DOI: https://doi.org/10.5153/ sro.548.

Ruíz Herrero, Jesús (2011): “Rendimiento académico y ambiente social”. Política y Sociedad, 48 (1), 155-174 (en línea). https://revistas.ucm.es/index.php/POSO/article/view/POSO1111130155A, consultado el 16 de abril de 2020.

Seller, Sam y Gale, Trevor (2011): "Mobility, Aspiration, Voice: A New Structure of Feeling for Student Equity in Higher Education". Critical Studies in Education, 52 (2), 115-134. DOI: https://doi.org/10.10 80/17508487.2011.572826.

Shavit, Yossi; Arum, Rivhard y Gamoran, Adam (eds.) (2007). Stratification in Higher Education: A Comparative Study. Stanford: Stanford University Press.

Shavit, Yossi y Blossfeld, Hans Peter (eds.) (1993). Persistent Inequality: Changing Educational Attainment in Thirteen Countries. Boulder: Westview Press.

Sieben, Inge; Huinink, Johannes y de Graaf, Paul (2001): "Family Background and Sibling Resemblance in Educational Attainment. Trends in the Former FRG, the Former GDR, and the Netherlands". European Sociological Review, 17 (4), 401-430. DOI: https://doi.org/10.1093/esr/17.4.401.

Taylor, Steven y Bogdan, Robert (1986). Introducción a los métodos cualitativos de investigación. La búsqueda de significados. Buenos Aires: Paidós

Tejedor, Francisco Javier y Caride, José Antonio (1988): "Influencia de las variables contextuales en el rendimiento académico". Revista de Educación, 287, 113-146 (en línea). https://dialnet.unirioja.es/servlet/articulo?codigo=18662, consultado el 23 de febrero de 2020 .

Treiman, Donald y Kam-bor, Yip (1989): "Educational and Occupational Attainment in 21 Countries" en Melvin L. Kohn (ed.): Cross-National Research in Sociology. Beverly Hills: Sage Publications.

Weber, Max (1969). Economía y sociedad. Esbozo de sociología comprensiva. México: Fondo de Cultura Económica.

Willis, Paul (2008): "Los soldados rasos de la modernidad. La dialéctica del consumo cultural y la escuela del siglo XX". Revista de Sociología de la Educación, 1 (3), 43-66 (en línea). https://dialnet.unirioja.es/ servlet/articulo?codigo $=2794281$, consultado el 3 de marzo de 2020 .

Willis, Paul (1988). Aprendiendo a trabajar. Cómo los chicos de clase obrera consiguen trabajos de clase obrera. Madrid: Akal. 


\section{Nota biográfica}

Javier Donoso-Bravo es Sociólogo, Universidad de Valparaíso, Chile. Magíster en Sociología Económica, Instituto de Altos Estudios Sociales (IDAES), Universidad Nacional de San Martín (UNSAM), Argentina. Doctorando en Ciencias Sociales de la Universidad de Buenos Aires (UBA), Argentina y Docente de la Escuela de Trabajo Social, Universidad de Valparaíso, Chile. 